\title{
A Multicellular Approach Forms a Significant Amount of Tissue-Engineered Small Intestine in the Mouse
}

\author{
Frédéric G. Sala, Ph.D., Jamil A. Matthews, M.D., Allison L. Speer, M.D., Yasuhiro Torashima, M.D., Ph.D., \\ Erik R. Barthel, M.D., Ph.D., and Tracy C. Grikscheit, M.D.
}

Tissue-engineered small intestine (TESI) has successfully been used to rescue Lewis rats after massive small bowel resection. In this study, we transitioned the technique to a mouse model, allowing investigation of the processes involved during TESI formation through the transgenic tools available in this species. This is a necessary step toward applying the technique to human therapy. Multicellular organoid units were derived from small intestines of transgenic mice and transplanted within the abdomen on biodegradable polymers. Immunofluorescence staining was used to characterize the cellular processes during TESI formation. We demonstrate the preservation of Lgr5- and DcamK11-positive cells, two putative intestinal stem cell populations, in proximity to their niche mesenchymal cells, the intestinal subepithelial myofibroblasts (ISEMFs), at the time of implantation. Maintenance of the relationship between ISEMF and crypt epithelium is observed during the growth of TESI. The engineered small intestine has an epithelium containing a differentiated epithelium next to an innervated muscularis. Lineage tracing demonstrates that all the essential components, including epithelium, muscularis, nerves, and some of the blood vessels, are of donor origin. This multicellular approach provides the necessary cell population to regenerate large amounts of intestinal tissue that could be used to treat short bowel syndrome.

\section{Introduction}

Chort bowel syndrome (SBS) results after a severe $\checkmark$ truncation of the native small intestine, usually after surgical resection of injured or necrotic regions of intestine associated with neonatal conditions such as malrotation with volvulus or necrotizing enterocolitis, a common condition of premature babies that is increasing in incidence as more premature babies survive. ${ }^{1-3}$ Although less common, SBS is also seen in adults suffering from Crohn's disease or from mesenteric infarction. ${ }^{4,5}$ Intestinal failure from SBS generally occurs with loss of approximately $50 \%-75 \%$ of the small intestine. ${ }^{6-8}$ The insufficient quantity of surface absorption results in a broad diversity of metabolic and physiologic disturbances, including fluid, nutrient, and weight loss. ${ }^{9}$ SBS may lead to liver failure, as prolonged intravenous alimentation is associated with cholestasis and progression to cirrhosis. The incidence of SBS is increasing, estimated at 24.5 per 100,000 live births in $2004,{ }^{10}$ with a $30 \% 5$ year mortality rate and annual costs/patient in Europe ranging from $\$ 100,000$ to 150,000 . $^{11}$ Cost estimates for pediatric patients with SBS in the United States have been widely variable, but the average estimate is about $\$ 1000 /$ day, without including multiple hospitalizations for catheter-related sepsis or even- tual liver or liver/small bowel transplant. Although the success rate of intestinal transplantation to treat SBS is improving, a recent review of 141 intestinal transplants in 123 children reveals a 3 -year patient survival rate of $60 \%{ }^{12}$ In addition to this low success rate, intestine transplantation, or multivisceral transplantation including the liver in cases where liver cirrhosis has progressed during treatment of the SBS, is limited by organ supply, histocompatibility matching, immunosuppression regimens, overall costs, and complexity. ${ }^{1-3}$ To reduce the heavy human and financial costs of intestinal failure resulting from SBS, tissue engineering and other stem cell-based therapies are attractive research targets.

Intestinal epithelium is composed of crypts and villi adjacent to a surrounding innervated muscularis responsible for peristalsis. In normal conditions, the intestinal mucosa is very dynamic and regenerates its entire epithelium lining every 3-7 days. This is achieved by the proliferation of intestinal epithelial stem/progenitor cells located at the base of the crypts. As the daughter cells migrate along the crypt/ villus axis, they differentiate into four specialized cell types: goblet cells, Paneth cells, enteroendocrine cells, and enterocytes. When they reach the top of the villus, they undergo apoptosis and are shed into the lumen of the intestine. Several putative intestinal epithelium stem cell markers have 
been proposed, including Bmi1 $1,{ }^{13} \operatorname{Lgr} 5,{ }^{14} \mathrm{CD} 133,{ }^{15}$ DcamKL- $1,{ }^{16}$ and Musashi-1. ${ }^{17}$ Lgr5 and CD133 mark four to eight crypt base columnar cells located in between the Paneth cells at the base of the crypts. In vitro studies with isolated Lgr5-positive cells have resulted in the generation of rudimentary "crypt-like structures" containing no mesenchyme, and an epithelial tissue bearing all four differentiated intestinal cell types. ${ }^{18} \mathrm{On}$ the other hand, Bmi1, DcamKL-1, and Musashi-1 stain cells located in position +4 , right above the Paneth cells. Subcutaneous implantation of DcamKL-1-positive cells formed spheroid-like structures with glandular epithelium. ${ }^{19}$ However, for actual human therapy, a meaningful quantity of vascularized full-thickness intestine must be generated, with a fully differentiated epithelium alongside an innervated muscularis.

The goal of tissue engineering is to create living replacement organs and tissues, with the proposed advantages of more exact physiology and durability related to cellular proliferation and autologous repair. In the rat model, we were able to engineer the entire gastrointestinal tract. ${ }^{20-26}$ Importantly, we demonstrated that tissue-engineered small intestine (TESI) had absorptive functions and could be used as an effective rescue therapy to treat SBS after massive small bowel resection. ${ }^{24}$ In patients, most causes of intestinal loss are mechanical or related to infection rather than intrinsic cellular defects, making an autologous strategy to generate TESI possible. As proof of principle, this technique was used to engineer autologous small intestine and stomach in a Yorkshire swine model; a 6week-old piglet is the same size as a newborn baby vulnerable to intestinal loss from necrotizing enterocolitis. ${ }^{27}$

Previous work using GFP transgenic mouse cells have demonstrated that tissue-engineered intestine could form in this species. ${ }^{28}$ Our study focuses on the cells involved during the formation of TESI in this new mouse model. We demonstrate that a multicellular approach, in which the mesenchymal-epithelial interactions are maintained, generates a tissue-engineered intestine that is morphologically comparable to the native intestine. In addition to forming larger amounts of fully differentiated intestinal epithelium, our resulting TESI has the muscle, nerves, and blood supply necessary with lineage tracing demonstrating the donor origin of these components. These elements are necessary for eventual use as a rescue therapy to treat human intestinal failure.

\section{Materials and Methods}

\section{TESI formation}

Organoid units (OUs) were procured from 2-week-old donor mice of both genders and loaded onto biodegradable polymers (see below). These polymers, loaded with OUs, were then implanted into the omentum of adult Nonobese Diabetic/Severe Combined Immunodeficiency (NOD/SCID) gamma host mice, wrapping the omentum in close contact with the polymer and securing them together with a single poliglecaprone 25 5-0 suture. For time course experiments, samples were harvested at 1 day, 3 days, 5 days, 7 days, 14 days, 4 weeks, and 6 weeks after implantation.

\section{OU production and implantation}

All animal protocols were carried out after protocol approval by the Childrens Hospital Los Angeles, IACUC.
Under sterile conditions, resected tissue was irrigated with cold Hanks balanced salt solution to clean luminal contents. The tissue was divided into full-thickness $2 \times 2 \mathrm{~mm}$ sections. These were washed twice in $4^{\circ} \mathrm{C}$ Hanks balanced salt solution, sedimented between washes, and then digested in $800 \mathrm{U} / \mathrm{mL}$ of collagenase type I (Worthington) and $0.12 \mathrm{mg} /$ $\mathrm{mL}$ of dispase (Gibco, Invitrogen). The digestion was stopped using cold 4\% Sorbitol (Sigma-Aldrich) and 10\% fetal bovine serum (Invitrogen), in high-glucose Dulbecco's modified Eagle's medium (Invitrogen). Large debris is excluded by removing the supernatant after digestion. The OUs in this supernatant are then isolated as separate from the single cells by centrifugation at $500 \mathrm{rpm}$ for $10 \mathrm{~min}$. The resulting pellet containing the OUs is resuspended in $10 \%$ fetal bovine serum and high-glucose Dulbecco's modified Eagle's medium. OU were loaded on the biodegradable scaffolds and directly implanted into the omentum of 3- to 6month-old mice. NOD/SCID gamma mice (The Jackson Laboratory) were used in order to avoid immune rejection of the implanted cells derived from the various backgrounds of transgenic mice. Just before implantation, the mice received a full-body irradiation (350 CGy) in order to reduce the immune reactivity still present in those mice. Alternatively, OUs were not implanted and collected in formalin for histology and immunohistochemistry.

\section{Polymer preparation}

Two-millimeter-thick nonwoven poly-glycolic acid (bulk density, $60 \mathrm{mg} / \mathrm{cm}^{3}$; porosity $>95 \%$ ) (Concordia Fibers) are formed on a glass mandril from flat felt $(4 \mathrm{~mm}$ long, $4 \mathrm{~mm}$ diameter) and sealed with 5\% poly-L-Lactic acid (Durect Corporation) in chloroform (Sigma-Aldrich). After sterilization with $100 \%$ ethanol, the polymers are coated with $0.4 \mathrm{mg} / \mathrm{mL}$ collagen type I solution (Sigma-Aldrich) for $20 \mathrm{~min}$ at $4^{\circ} \mathrm{C}$, rinsed with phosphate-buffered saline, and stored dried at room temperature. Polymers were stored in a desiccator, in order to avoid premature hydrolysis and degradation of the polymer. Polymers were loaded with OUs $\left(25.4 \times 10^{6}\left[ \pm 1.5 \times 10^{6}\right]\right.$ total cells $)$.

\section{Transgenic mice}

The $\operatorname{actin}^{G F P}$ mice $^{29}$ were maintained on a C57BL/6 background (a kind gift from Dr. Wagers). Lgr $5^{\text {egfp }}$ mice were maintained on a C57BL/6 background (The Jackson Laboratory). NOD/SCID gamma mice were obtained from The Jackson Laboratory.

\section{Immunostaining}

The TESI was fixed overnight in $10 \%$ formalin and processed for paraffin embedding. The samples were sectioned at $5 \mu \mathrm{m}$ thickness. Antigen was retrieved by boiling the slides using a microwave for $20 \mathrm{~min}$ in $10 \mathrm{mM} \mathrm{Na}$-Citrate ( $\mathrm{pH}$ 6.0). The slides were incubated overnight at $4^{\circ} \mathrm{C}$ with the following primary antibodies: lysozyme (1/100; DakoCytomation), chromogranin A (1/100; ABcam), villin (1/100; Santa Cruz), E-cadherin (1/100; Santa Cruz), pan-Cytokeratin (1/50; Sigma-Aldrich) proliferating cell nuclear antigen (PCNA) (1/100; Vector), DcamKL-1 (1/50; ABgent), PECAM (1/100; NeoMarkers), desmin (1/50; DakoCytomation), smooth muscle actin (SMA) (1/300; Sigma-Aldrich), smooth muscle myosin 
heavy chain (1/100; ABcam), S-100 (1/50; SigmaAldrich), Neurofilament (1/50; Sigma-Aldrich), and GFP (1/ 200; ABcam). Cy3-, Cy5-, or FITC-conjugated secondary antibodies were used, and slides were mounted with Vectashield containing DAPI (Vector). The pictures were acquired using an upright fluorescent microscope (Leica DM5500). All the stainings were performed in at least three different specimens.

\section{Histological staining}

Five-micrometer cross-sections were stained with hematoxylin and eosin according to standard procedures or with alcian blue as described in www.ihcworld.com/protocols/ special_stains/alcian_blue.htm

\section{Quantification of GFP-positive donor cells}

TESI were generated from OUs derived from actin ${ }^{G F P}$ intestine and implanted into irradiated NOD/SCID gamma mice in two separate experiments. The implants were harvested at specific time points, $\mathrm{T}=0(n=3)$, at 2 weeks $(n=4)$, 4 weeks $(n=5)$, and 6 weeks $(n=5)$. The tissues were digested to single cells using $1600 \mathrm{U} / \mathrm{mL}$ of collagenase type I (Worthingthon) for $30 \mathrm{~min}$ at $37^{\circ} \mathrm{C}$. The total number of cells for each sample was quantified using a hemacytometer (Hausser Sceintific). Percentage of GFP-positive cells was evaluated for each sample by flow cytometry. Native intestine from NOD/SCID mice was used as GFP-negative tissue to set the gates. Of note, the percentage of GFP-positive cells in the native intestine was only 50\%. The number of GFPpositive cells for each harvested tissue was determined and plotted on a graph (Fig. 8). Statistical analysis was performed using a two-tail, two-sample unequal variance, Student's t-test (Excel; Microsoft) between samples at $\mathrm{T}=0$ and 6 weeks.

\section{Quantification of Lgr5-positive and differentiated epithelial cells}

Sections from TESI and native intestine were stained using specific markers for each cell type. The goblet cells were marked by alcian blue staining. The Paneth cells were revealed using Lysozyme immunostaining. The enteroendocrine cells were stained with a Chromogranin A antibody. Paneth cells were counted per crypt. Goblet cells and enteroendocrine cells were counted per hemi crypt/villus axis.
A total of 25 crypts or hemi crypt/villus axis was counted in five different TESI (5 per TESI) and a total of 15 in three different native intestines (5 per intestine). Statistical analysis was performed using a two-tailed, two-sample unequal variance, Student's $t$-test (Excel; Microsoft) for each cell type.

The Lgr5-positive cells in Lgr5 $5^{\text {egfp }}$ engineered intestine were stained using an EGFP antibody. Eight crypts total were counted in two different TESI.

\section{Results}

\section{TESI grows as an expanding globe organized} with an outer muscularis and an inner intestinal epithelium

A cylindrical biodegradable polymer scaffold made of a $2 \mathrm{~mm}$ nonwoven polyglyoclic acid, $4 \mathrm{~mm}$ long and $4 \mathrm{~mm}$ in diameter (Fig. 1A), is seeded with OUs generated from the small intestine of 2-week-old mouse pups. The average number of cells implanted into the scaffold in form of OUs is about $3.07 \times 10^{6}\left( \pm 0.5 \times 10^{6}\right)$. This corresponds to about two scaffolds per length of neonatal intestine. Four weeks after implantation into the omentum of irradiated NOD/SCID gamma mice, the implants had grown to form spherical tissue-engineered intestine, with a diameter about twice the size of the original implanted polymer (Fig. 1A). After embedding and sectioning, a low magnification picture of hematoxylin and eosin staining shows a large quantity of mucosal epithelium lining one or several lumens (Fig. 1B). The bottom left corner of the picture shows pancreatic tissue from the host that had attached to the external wall of the TESI (Fig. 1B). A high magnification picture of this mucosa demonstrates normal architecture of the glands of Lieberkühn, with crypts and villi. The formed epithelium is a simple columnar epithelium with identifiable goblet cells along the crypt/villus axis and abundant Paneth cells at the base of the crypts (Fig. 1C). TESI was successfully generated from donor tissue of different backgrounds (C57B16, CD1, and mixed background) implanted into NOD/SCID gamma mice and from C57Bl6 donor tissue into C57B16 host animals (data not shown).

In this murine model, our success rate in regenerating a new epithelium was $89 \%$ (39 out of 44 implants). The heterogeneity of the generated epithelium ranged from a flat epithelium with no differentiated cells (1 out of 44 implants),
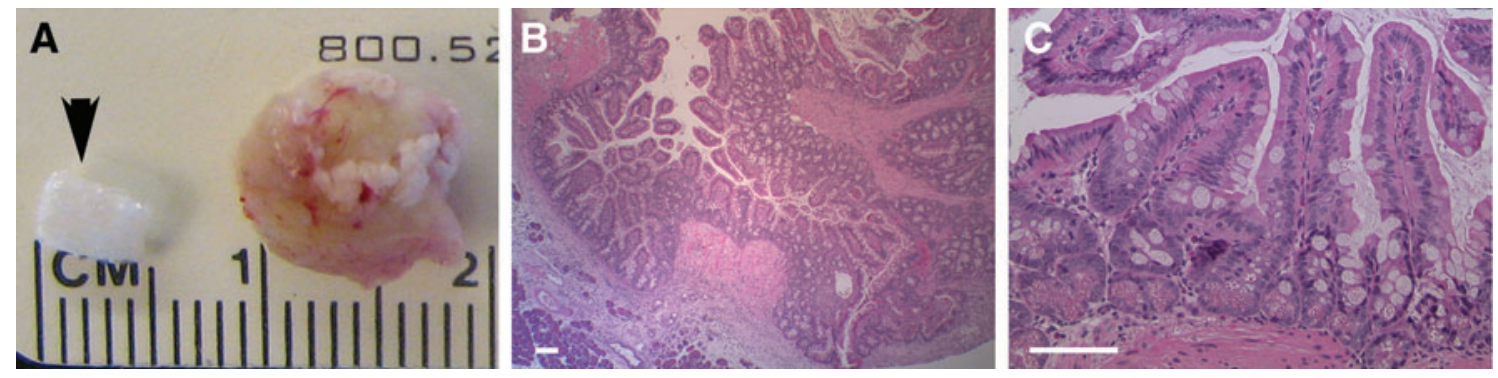

FIG. 1. Morphology of TESI in the mouse model. (A) Four weeks after implantation, the tissue-engineered intestine formed a sphere about twice the size of the initial implanted polymer (arrow). (B) Hematoxylin and eosin staining at low magnification demonstrates a large amount of continuous mucosa lining the lumen of the TESI. (C) At higher magnification, the mucosa of the TESI is composed of a simple columnar epithelium forming crypt and villus structures. Goblet cells, along the crypt and villus axis, and Paneth cells, at the base of the crypts, can be identified. Scale bar: $40.0 \mu \mathrm{m}$. TESI, tissue-engineered small intestine. Color images available online at www.liebertonline.com/tea 
to flat epithelium with crypts only (15 out of 44 implants), to fully formed crypts and villi surrounded by flat epithelium (23 out of 44 implants) (data not shown). When only crypts, or crypts and villi were observed, we also noticed the presence of differentiated cells. Eighty six percent (38 out of 44 implants) of the implants demonstrated epithelial differentiation (presence of Goblet cells and/or Paneth cells). All the samples that generated an epithelium also grew a muscularis more or less developed.

\section{TESI possesses all four differentiated epithelial cell types present in native small intestine}

The presence and normal location of all four differentiated epithelial cell types in the TESI was confirmed in five different specimens using immunohistology techniques and compared to native small intestine. Goblet cells, which produce mucus, were identified by alcian blue staining in large numbers (Fig. 2A). An average of $9( \pm 3)$ goblet cells was counted by hemi-crypt/villus axis in TESI. This number was higher ( $p$-value $\leq 0.05)$ than the number of goblet cells present in native ileum $(7 \pm 3)$. Paneth cells, which contribute to the regulation of the intestinal flora, are normally located at the base of the crypts in native small intestine and stained positive for lysozyme. Paneth cells are similarly identified in the TESI (Fig. 2B). We observed a slightly higher number of Paneth cells in TESI ( $7 \pm 2$ per crypt) compared to native intestine $(6 \pm 1)$, although this difference was not statically significant. Enteroendocrine cells, which produce hormones necessary for the control of the digestion process, are revealed using Chromogranin A antibody in both native small intestine and TESI at a rate of 1 to 2 cells per hemi crypt/ villus axis (Fig. 2C). Finally, the microvilli of the absorptive enterocytes are stained using a villin antibody in the native small intestine and in the TESI (Fig. 2D).

\section{TESI contains an innervated muscularis}

TESI has an SMA-positive, Desmin-positive muscularis (Fig. 3A). In order to confirm the maturity of the muscle cells, the presence of smooth muscle myosin heavy chain was demonstrated by co-immunostaining with SMA and Desmin (Fig. 3B). However, the fibers do not recapitulate the circular and longitudinal organization observed in the native intestine (Fig. 3A, B). In the native intestine, the muscularis is innervated by the ganglion cells of Meissner's plexus, located in the submucosa, and of Auerbach's plexus, located between the circular and longitudinal layers of the muscularis. Although at 4 weeks the double-layered muscularis is not reproduced in the TESI, S100 (Fig. 3C) and Neurofilament (Fig. 3D)-positive ganglion cells were observed in the submucosa (white arrows) and/or in between the muscular fibers (yellow arrows) roughly correlating to Meissner's and Auerbach's plexuses.

\section{TESI possesses features of an intact stem cell niche}

The intestinal epithelium renews itself every 3-7 days. The epithelium regenerates from stem cells located toward the base of the crypts. Those stem cells are positioned in a stem cell niche that is principally composed of mesenchymal intestinal subepithelial myofibroblasts (ISEMFs). Two distinct cell populations have recently been proposed to be adult intestinal stem cells. Lgr5 stains 4-8 cells at the base of each crypt, in between the Paneth cells. ${ }^{14}$ Another marker, DcamKL-1, stains one single cell per crypt at the position +4 , just above the Paneth cells. ${ }^{16}$ Those cells are described to be in a quiescent state and therefore negative for PCNA staining. ${ }^{16}$ Using the small intestine of the transgenic reporter line Lgr5 ${ }^{\text {EGFP14 }}$ as donor cells to generate OUs, we observed Lgr5positive cells in TESIs generated with this transgenic line in a location comparable to native tissue (Fig. 4A). Interestingly, we observed between 12 and 19 Lgr5-positive cells per crypt in the tissue-engineered intestine. This is double the amount that Barker et al. reported in the native intestine. ${ }^{14}$

Double immunofluorescence staining for both DcamKL-1 and PCNA demonstrates the presence of quiescent DcamKL1-positive cells in the native small intestine and the TESI (Fig. 4B). In addition, ISEMF, positively stained for SMA and negative for Desmin, are seen in their appropriate location
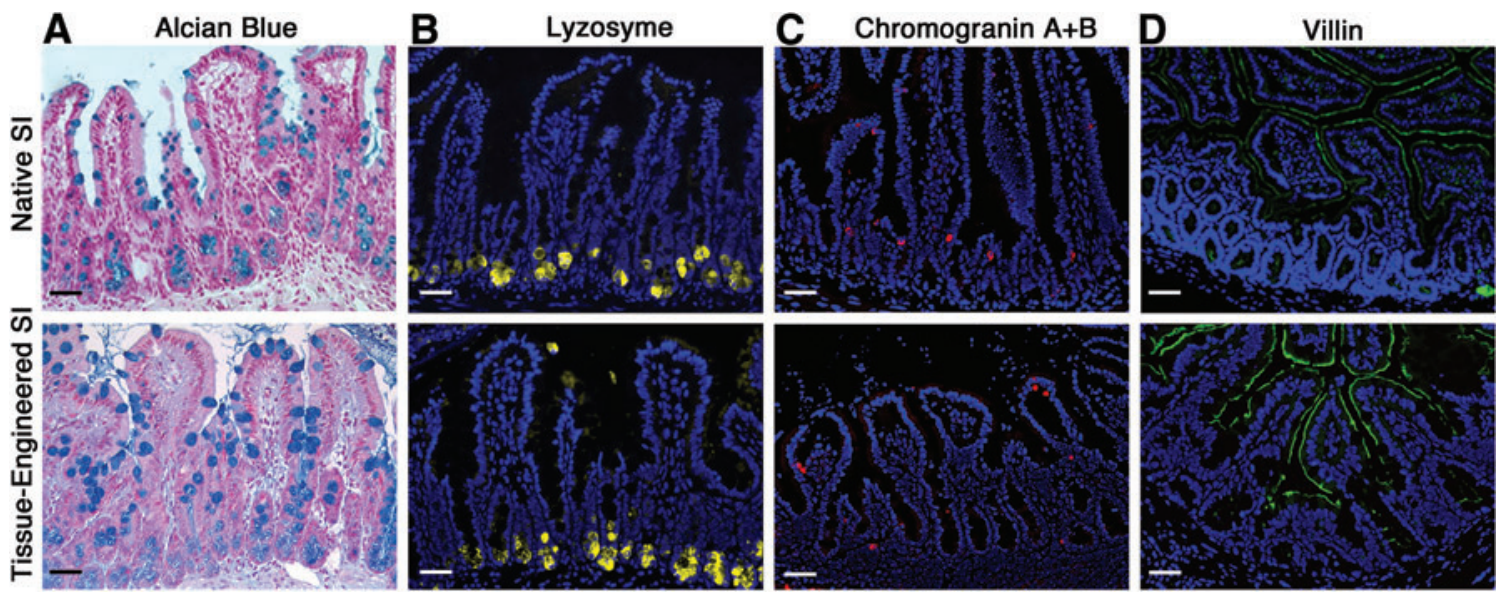

FIG. 2. TESI demonstrates normal epithelial differentiation. (A) Alcian blue staining of the goblet cells in the native and the TESI, respectively. (B) Lysozyme staining demonstrates the presence of Paneth cells in their crypt location in tissueengineered intestine and native intestine. (C) Chromogranin A staining identifies enteroendocrine cells in both native and tissue-engineered intestine. (D) The brush border of the enterocytes is stained using villin-specific antibody in both tissues. Scale bar: $40.0 \mu \mathrm{m}$. Color images available online at www.liebertonline.com/tea 

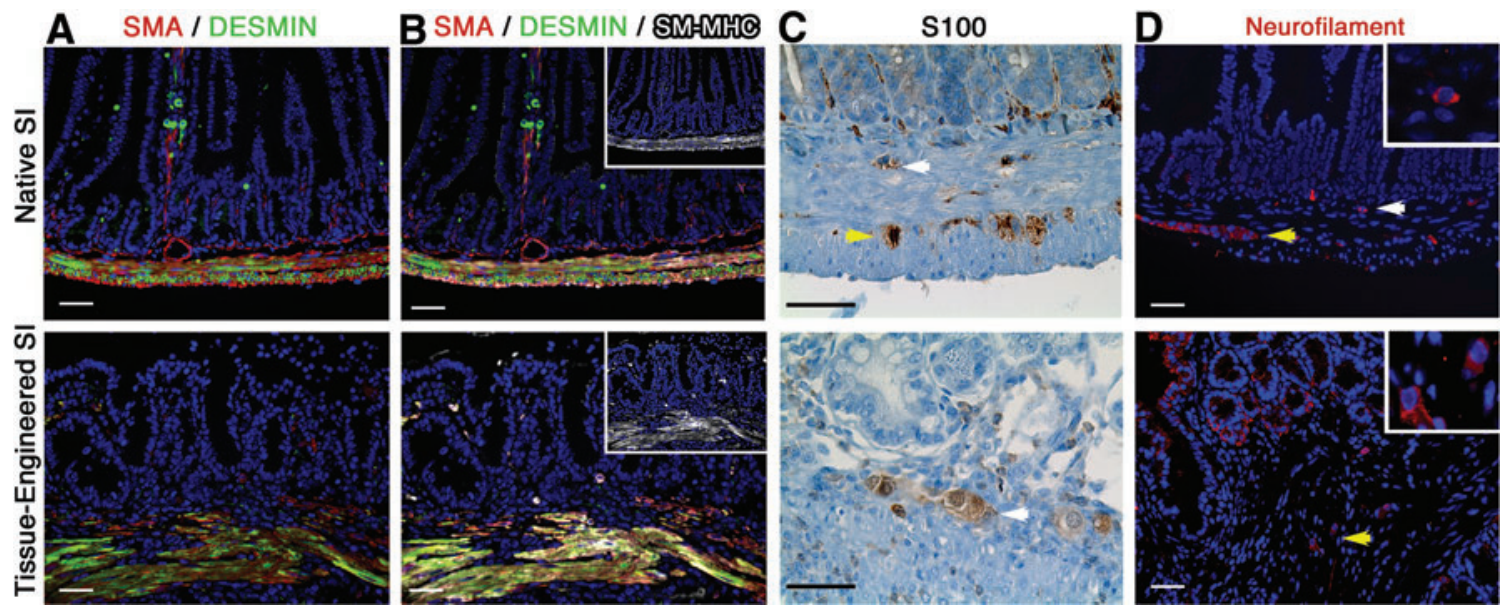

FIG. 3. TESI demonstrates an innervated muscularis. (A) The muscularis, positive for both SMA and Desmin, although present in the engineered intestine, did not recapitulate the transversal and circular organization normally observed in the native small intestine. (B) SM-MHC (insets in B) co-localized with SMA and Desmin demonstrating the maturity of the smooth muscle fibers. (C) S100 and (D) Neurofilament immunostainings identify typical round ganglion cells (insets in D) in the submucosa (white arrows) and in between the muscle fibers (yellow arrows) of native and TESI roughly corresponding to both Meissner and Auerbach's plexi. Scale bar: $40.0 \mu \mathrm{m}$. SMA, smooth muscle actin; SM-MHC, smooth muscle myosin heavy chain. Color images available online at www.liebertonline.com/tea

just below the base of the crypt epithelium in both native and engineered small intestine (Fig. 4C).

The time course for the formation of TESI shows that the niche is preserved throughout tissue growth

To characterize the first events of TESI formation, we harvested TESI on serial days after implantation. One day after implantation, we observed that many of the implanted cells are dead as evidenced by the lack of nuclear staining with hematoxylin (green arrows in Fig. 5A). The viable cells (black arrow) are "floating" in between the fibers of the polymers toward the periphery of the polymer (Fig. 5A). After 3 days, a few OUs are discernible in the outer layer of the polymer, right at the contact point with the host omentum, which will be the major source of vascular ingrowth.
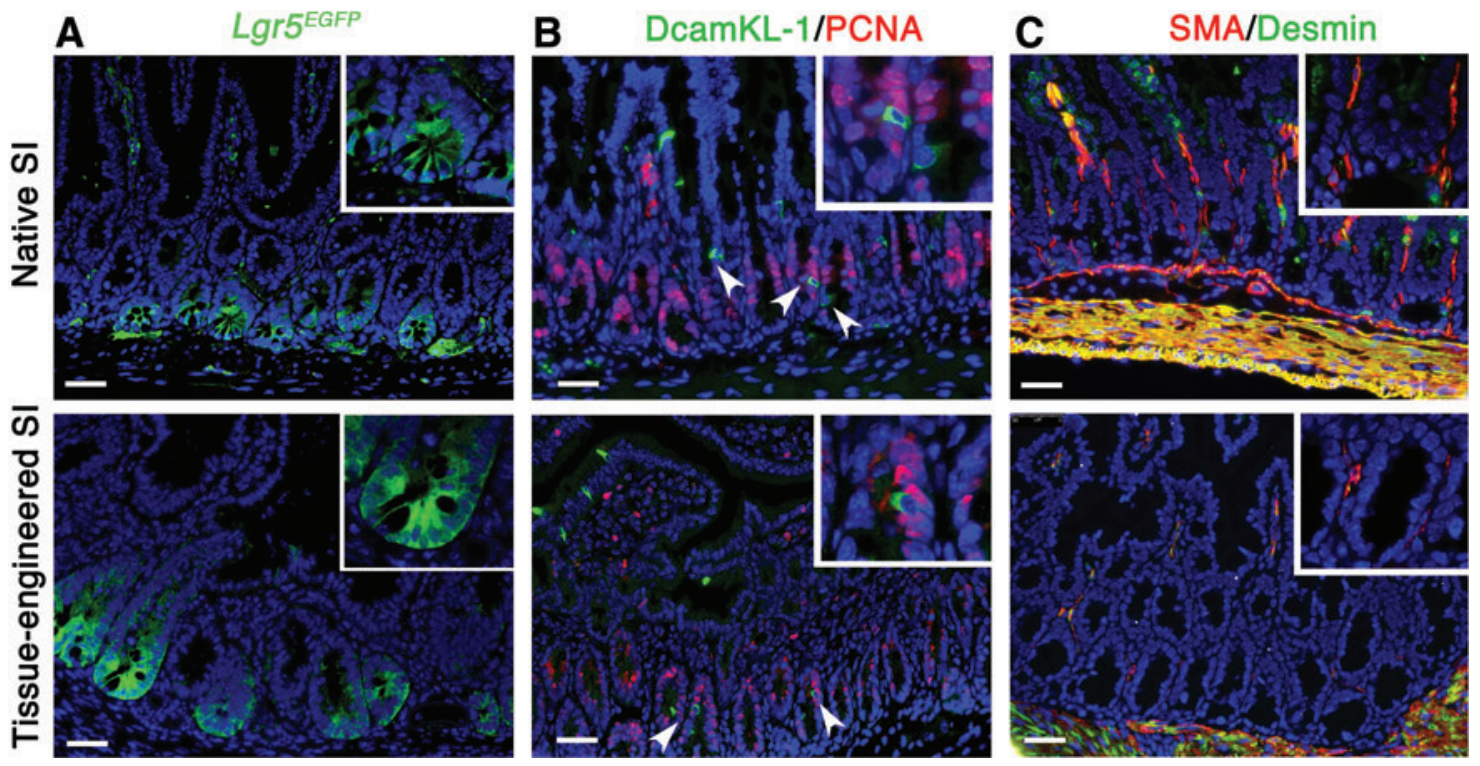

FIG. 4. Components of an intestinal stem cell niche are identified in native and engineered small intestine at 4 weeks. Both Lgr5 and DcamKL-1 are potential markers for the intestinal epithelial stem cells. (A) Using $\operatorname{Lgr} 5^{E G F P}$ intestine as donor tissue to generate TESI in NOD/SCID gamma mice, Lgr5-positive cells are identified at the base of the crypts of TESI similar to the native intestine donor tissue. (B) DcamKL-1 staining, identifying a putative stem cell in position +4 , demonstrates the presence of these cells in both native and engineered tissue. Those cells are negative for PCNA, confirming their quiescence (arrowheads and insets). (C) ISEMF, mesenchymal cells of the stem cell niche, are characterized as positive for SMA and negative for Desmin in both native and engineered intestine (insets). Smooth muscle cells, positive for both markers, appear in yellow. Scale bar: $40.0 \mu \mathrm{m}$. ISEMF, intestinal subepithelial myofibroblasts. PCNA, proliferating cell nuclear antigen. Color images available online at www.liebertonline.com/tea 

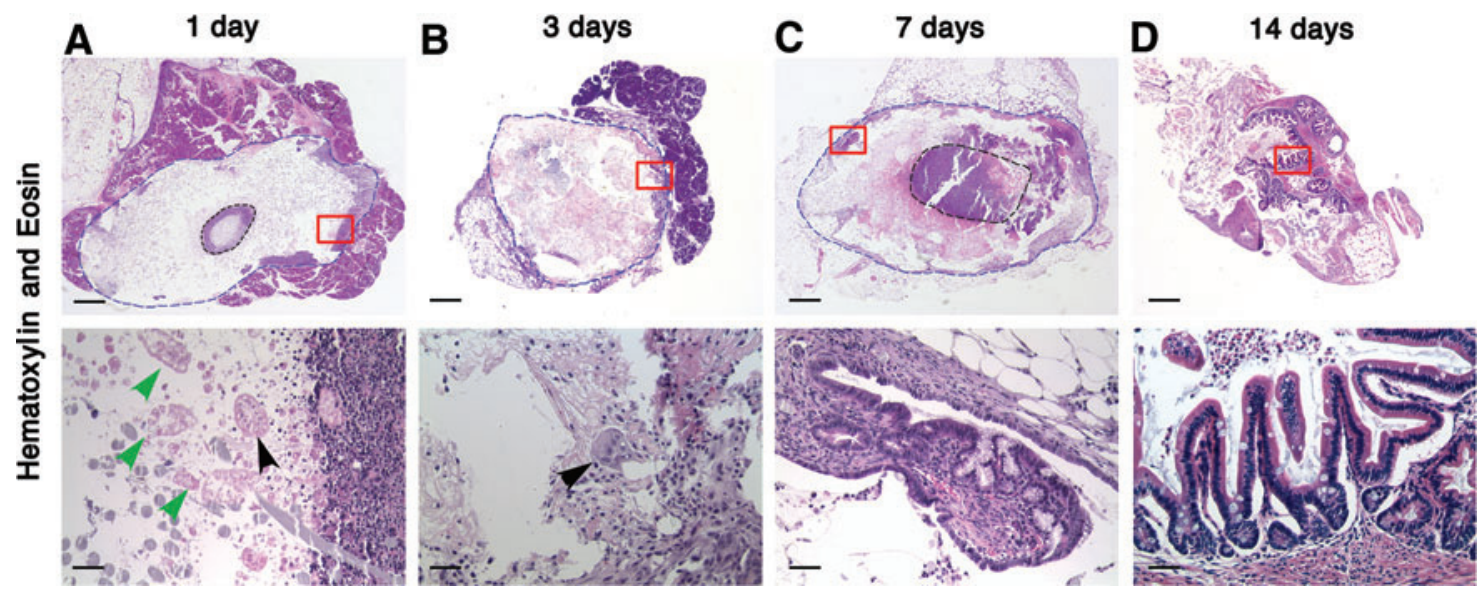

FIG. 5. Time course of the development of TESI. TESI is shown at low magnification looking down the lumen of the tubular polymer at sequential time points and at high magnification at the area marked with the red box for cellular detail. (A) One day after implantation, organoid units that are still viable (black arrowheads) are floating in between the fibers of the polymer. The green arrowheads show dead organoid units. (B) At 3 days, the organoid units (arrowhead) are surrounded by connective tissue. (C) At 7 days, a flat epithelium develops with rudimentary crypts. (D) By 14 days, a complete epithelium forms with crypts and villi. Scale bar: low magnification: $4.0 \mu \mathrm{m}$; high magnification: $40.0 \mu \mathrm{m}$. Color images available online at www.liebertonline.com/tea

At this time, the OUs are surrounded by connective tissue from host origin (GFP negative) (Figs. 5B and 6B). No differentiated cells were observed at that time (data not shown). As early as 7 days, we observe a flat epithelium with a few crypts forming at the edge of the polymer, in proximity to the omental tissue (Fig. 5C). The goblet cells start to differentiate.
By 14 days, the flat epithelium has proliferated and developed into a normal-appearing intestinal epithelium with an identifiable crypt/villus axis (Fig. 5D). At that time, all four differentiated epithelial cell type are present. By 4 weeks, the quantity of engineered epithelium has continued to increase, with the addition of mesenchymal structures (Figs. 1-4).
FIG. 6. Epithelial-mesenchymal interactions are preserved in the implanted organoid units and during TESI formation.

(A) Double staining for E-cad, marking epithelial cells, and SMA staining the ISEMF demonstrate the mesenchymal and epithelial heterogeneity of the organoid units and the maintenance of the epithelial mesenchymal spatial relationship. DcamKL-1- and Lgr5-positive cells are found in close proximity to SMA-positive ISEMF, suggesting that the intact stem cell niche is preserved. (B) Actin GFP donor cells transplanted into NOD/SCID gamma mice demonstrate that epithelial-mesenchymal interactions are still present 3 days after implantation, as shown by the co-immunolocalization of GFP with E-cad and SMA. Note that the GFP-implanted cells, still in form
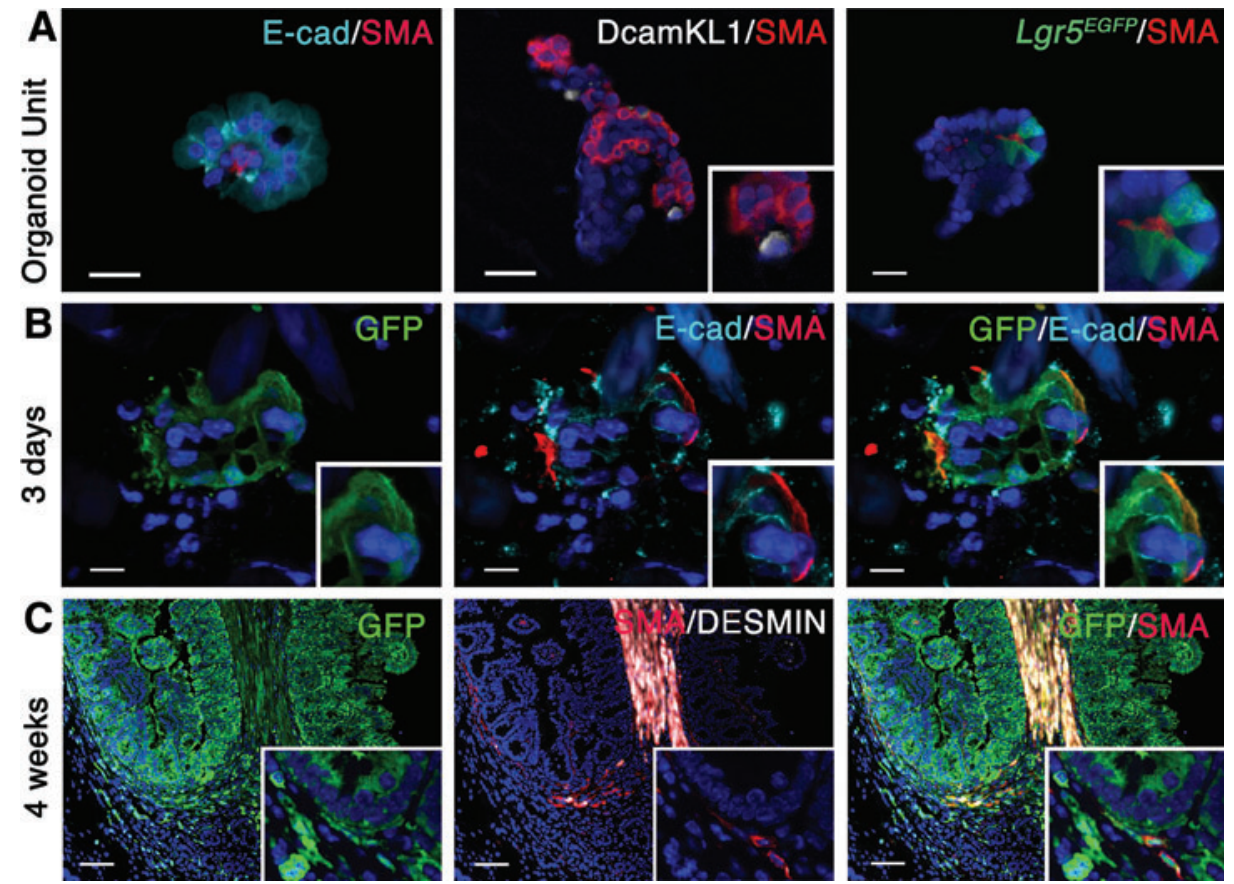
of organoid units, are sur-

rounded by GFP-negative cells (DAPI staining of the nuclei). The larger blue forms are shards of polymer that are not yet hydrolyzed. (C) Low magnification demonstrates the donor and host contribution to the TESI at 4 weeks. The entire epithelium is GFP positive. The mesenchymal compartment is from both donor and host origin. However, the spatial relationship between crypt epithelium and ISEMF is preserved in a fully grown tissue-engineered intestine after implantation (insets). Scale Bar: (A) $20.0 \mu \mathrm{m}$; (B) $10.0 \mu \mathrm{m}$; (C) $80.0 \mu \mathrm{m}$. E-cad, E-cadherin. Color images available online at www .liebertonline.com/tea 

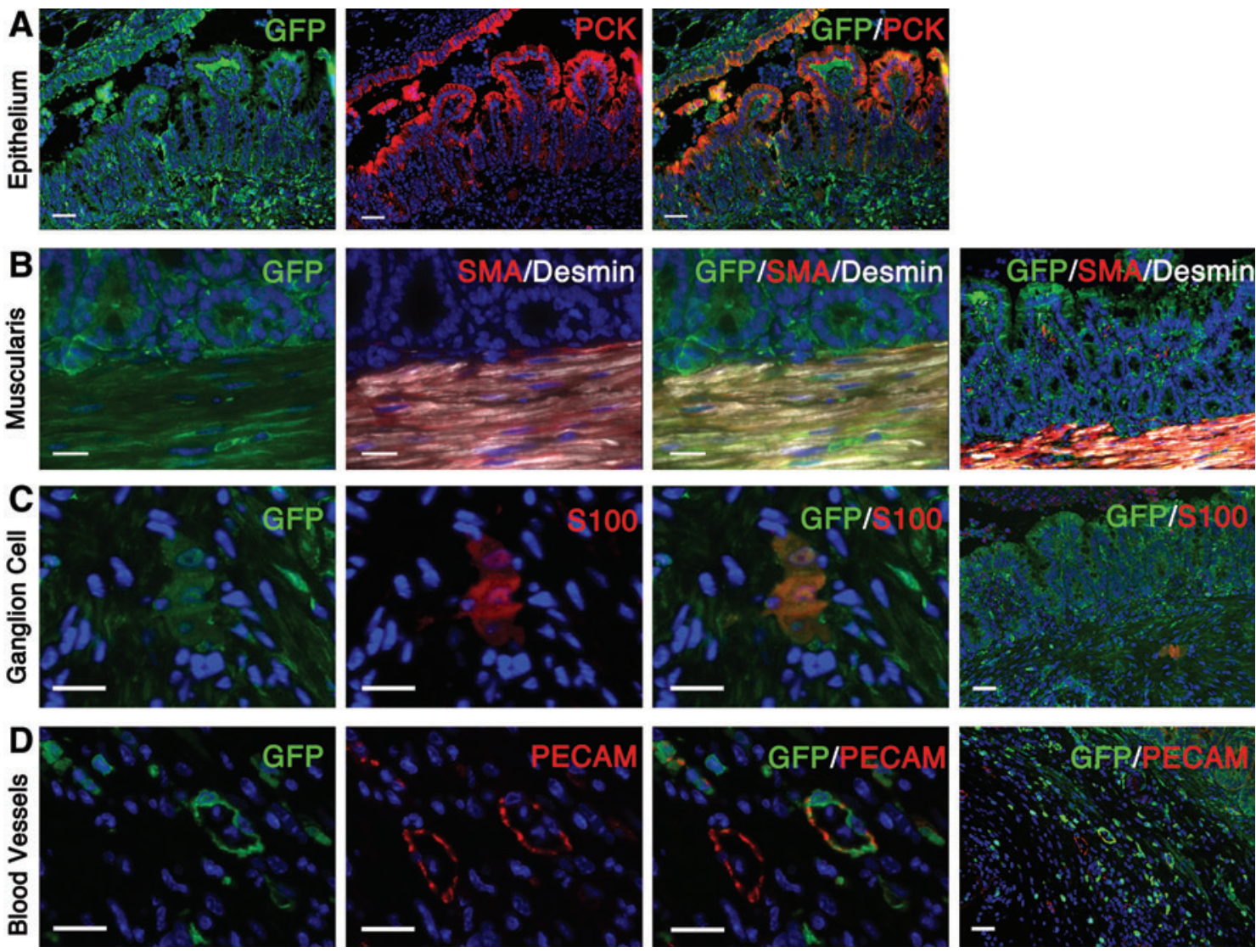

FIG. 7. Lineage tracing of critical epithelial and mesenchymal cell types of TESI. TESI generated from GFP-positive donor cells and implanted into a NOD/SCID gamma host demonstrates the donor origin of all the different cell types. (A) GFP and PCK co-localization confirms that the entire epithelium is from donor origin. (B) The muscularis, positive for GFP, SMA, and Desmin, derives from the implanted cells. (C) Co-immunolocalization of GFP and S100 demonstrates the donor origin of the ganglion cells. (D) Some blood vessels, positive for PECAM, but not all, are also positive for GFP, demonstrating a donor contribution to the vasculature. Scale bar: (A) $40.0 \mu \mathrm{m}$; (B-D) high magnification, $20.0 \mu \mathrm{m}$; low magnification, $80.0 \mu \mathrm{m}$. PCK, pan Cytokeratin. Color images available online at www.liebertonline.com/tea

\section{Mesenchymal and epithelial interactions are preserved during the growth of TESI}

OUs are multicellular clusters of cells of different shape and size. They are derived from and contain all the cell types of full-thickness intestine (data not shown). The majority of the OUs, but not all, are composed of mesenchymal and epithelial cells as shown in Figure 6A by an E-cadherin and SMA double immunostaining. Double staining for DcamKL1 and SMA demonstrates the proximity of the DcamKL-1positive cells and ISEMF. Likewise, Lgr5-positive cells can be found adjoining intestinal subepithial myofibroblasts in OUs derived from $\operatorname{Lgr} 5^{E G F P}$ small intestine.

In order to follow the implanted cells during the formation of the TESI, we used actin ${ }^{G F P}$ transgenic mice for donor tissue and implanted the cells into the omentum of adult NOD/ SCID gamma mice. Co-immunolocalization of E-cadherin and SMA with GFP demonstrates that the mesenchymalepithelial proximity initially observed in the OUs is preserved 3 days after implantation (Fig. 6B), within OUs as described previously in Figure 5B. The GFP-positive ISEMF are still identified adjacent to GFP-positive intestinal epithelium in fully grown TESI 4 weeks after implantation (insert in Fig. 6C). Interestingly, while the epithelium is positive for GFP, only parts of the mesenchymal compartment are derived from the implanted GFP-positive cells (Fig. 6C).

\section{All the major mesenchymal and epithelial cell types forming the TESI derive from the implanted cells}

To confirm the origin of each cell type composing the engineered tissue, OUs derived from actin ${ }^{G F P}$ small intestine were implanted into the omentum of adult NOD/SCID gamma mice. After 4 weeks of implantation, co-immunostaining for GFP and pan-Cytokeratin confirmed that the entire epithelium is from donor origin (Fig. 7A). These results were further verified by transplanting wild-type cells into actin $^{G F P}$ mice as well as by tracing the absence of the $\mathrm{Y}$ chromosome after implanting female tissue into male hosts (data not shown). Co-immunolocalization of GFP and specific markers demonstrated that all of the basic components of the TESI derive from the transplanted GFP-positive multicellular OUs. The muscularis was found to be positive for desmin, SMA, and GFP (Fig. 7B). S100-positive ganglion cells were also GFP positive (Fig. 7C). Finally, some blood vessels stained with PECAM were also GFP positive. These blood vessels are adjacent to GFP-negative blood vessels, perhaps 
indicating the site of anastomosis between the host and donor capillary systems (Fig. 7D).

\section{Quantification of the number of GFP-positive cells in the engineered tissue reveals that TESI grows beyond the original number of implanted cells}

To demonstrate that we are able to generate a TESI bigger than the original initial donor tissue, we implanted actin ${ }^{\text {GFP }}$ OUs into adult irradiated NOD/SCID gamma mice and harvested the tissues at various time points. After dissociation to a single cell suspension, the total number of cells per sample was counted. The percentage of GFP-positive cells was determined using a flow cytometer.

We observed a decrease in the number of GFP-positive cells at 2 weeks followed by progressive increase at 4 and 6 weeks (Fig. 8). Immediately after implantation, the average number of GFP-positive cells was $1.23 \times 10^{6}\left( \pm 0.08 \times 10^{6}\right)$, corresponding to $40.6 \%$ of the total number of cells $\left(3.07 \times 10^{6} \pm 0.5 \times 10^{6}\right)$, including the omental tissue of the host mouse. At 2 weeks, we observed a strong reduction of the number of GFP-positive cells $\left(0.26 \times 10^{6} \pm 0.2 \times 10^{6}\right)$, confirming our previous observation that most of the cells die within the first few days after implantation. Accordingly, the percentage of GFP cells also decreased to $17 \%$. However, by 4 weeks, the TESI regained their original size in average $\left(1.34 \times 10^{6} \pm 0.97 \times 10^{6}\right)$, corresponding to $23.6 \%$ of the total number of cells. The engineered tissues kept growing at 6 weeks; the average number of GFP-positive cells was

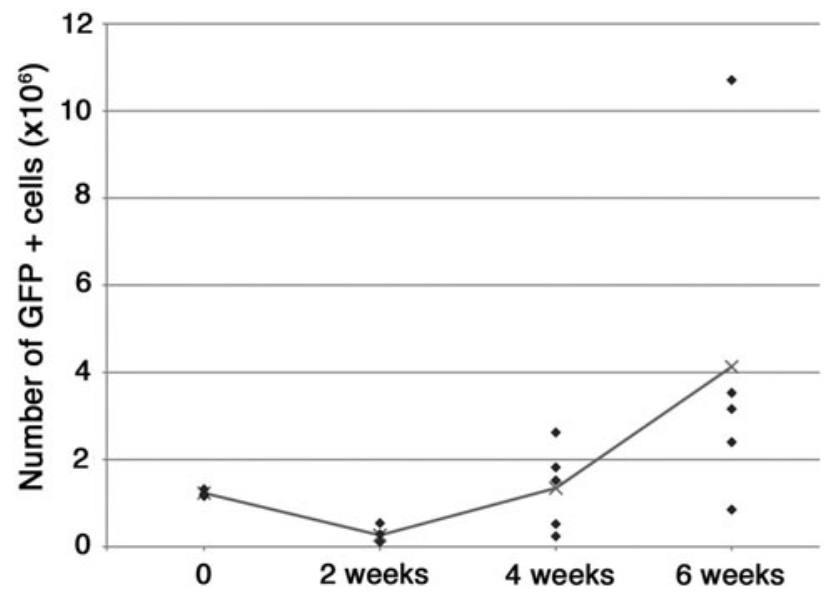

FIG. 8. Quantification of the number of GFP-positive cells forming the TESI over time. Actin ${ }^{G F P}$ organoid units were implanted into NOD/SCID gamma mice. Immediately after implantation $(n=3)$, at 2 weeks $(n=4)$, at 4 weeks $(n=5)$, and at 6 weeks $(n=5)$, the samples were harvested and digested to a single-cell suspension. Total numbers of cells were counted and percentages of GFP-positive cells were assessed using a flow cytometer. Each lozenge represents the value obtained for each sample. The line corresponds to the average value at each time point. We noticed an initial decrease in the number of positive cells in the first 2 weeks after implantation. However, the average quantity of GFP-positive cells increased at 4 weeks, although the variation between samples is important. By 6 weeks, the average number of GFP cells was three times the initial quantity used for implantation. This increase was not statistically significant when compared between $\mathrm{T}=0$ and 6 weeks samples.
$4.13 \times 10^{6}\left( \pm 3.8 \times 10^{6}\right)$, corresponding to more than $3 \times$ the original number of GFP-implanted cells. The percentage of GFP cells remains stable (21.4\%) (Fig. 8). Three samples (two at 4 weeks and one at 6 weeks) did not grow back to the original number of implanted cells. We speculate that those implantations failed. This is in accordance with our success rate of $89 \%$. Although there is an obvious trend toward growth beyond the number of implanted cells, the difference observed between $\mathrm{T}=0$ and 6 weeks was not statistically significant.

\section{Discussion}

The translation of this technique to a model that lends itself to mechanistic studies because of the wide variety of investigational tools available in the mouse is an advance. The implantation of OUs on a biodegradable polymer into the omentum allows the generation of TESI in an anatomic location, containing a large amount of intestinal mucosa as well as the mesenchymal components of intestine, including muscle and nerve. Using appropriate markers, we confirmed that the engineered tissue recapitulates the morphology of the native intestine with a fully differentiated epithelium adjacent to an innervated muscularis. This engineered tissue also bears both Lgr5- or DcamKL-1-positive cells, two putative intestinal stem cell populations, as well as the mesenchymal component of the stem cell niche, the ISEMFs, in their accustomed location.

OUs are heterogeneous clusters of cells that can contain both epithelial and mesenchymal cells, and in particular, some OUs contain either Lgr5- or DcamKL-1-positive cells adjacent to the mesenchymal ISEMF. The mesenchymal epithelial proximity, conserved in the OUs, is also seen during the growth of the TESI. Lineage tracing, which was not possible in previous tissue-engineered intestine models, demonstrates that all the essential components of the engineered intestine, epithelium, muscularis, nerves, and part of the blood supply are derived from the implanted cells.

Immediately after implantation, the majority of the implanted cells die. This is probably multifactorial. Because the OUs are generated from full-thickness intestine, the differentiated epithelial cells that no longer have the capacity to proliferate likely undergo apoptosis. Indeed, 3 days after implantation, we were unable to identify any differentiated epithelial cells (data not shown). These data suggest that the engineered tissue regenerates only from the implanted undifferentiated progenitor/stem cells. It is also probable that during the engineering process, some cells lose their anchorage to the extracellular matrix or neighboring cells and therefore undergo anoikis. Moreover, before the tissue completes angiogenesis and/or vasculogenesis, the implanted cells survive by imbibition of nutrients. Therefore, the cells located in the middle of the polymer may not receive adequate nutrients and oxygen to survive. This is supported by our observation that the regeneration of the tissue is initiated by a few OUs located on the outside layer of the polymer, in proximity to the omentum.

As early as 7 days after implantation, a flat epithelium and rudimentary crypt structure develops from the OUs that survive. By 14 days, crypt-villus structures are observed with a flat epithelium at each end of the epithelium. This process of formation is reminiscent of a wound healing process 
rather than a developmental process. After injury of the native intestine, an undifferentiated flat epithelium, originating from the edge of the wound, covers the exposed mesenchyme. This is followed by the invagination of new crypts and finally by the formation of villi. ${ }^{30}$ It is interesting to note that the number of Lgr5-positive cells as well as the number of differentiated epithelial cells was overall higher than what is found in native ileum. Similar findings have been observed in regenerating intestine, suggesting that the engineered intestine is still regenerating 4 weeks after implantation. ${ }^{31}$ Further investigation of the molecular and cellular mechanisms of TESI formation may help to identify mechanisms of tissue repair and vice-versa.

It is known that the intestinal epithelial stem cells survive in a mesenchymal cell niche toward the base of the intestinal crypt. Existing in a subepithelial location from esophagus to large intestine, ISEMFs are key mesenchymal cells supporting the intestinal stem cell and a major source of instructive signals to the intestinal epithelium. Beyond fetal development, a balanced gut homeostasis and repair process depends on mesenchyme-epithelial cross talk. ${ }^{32-34}$ Evidence for the preservation of an intact, populated stem cell niche in fully formed TESI includes the demonstration of a differentiated epithelium at 4 weeks, allowing for a number of rounds of the customary epithelial regeneration that occurs every 3-7 days. Although the roles of Lgr5- or DcamKL-1positive cells are still being defined, demonstration of the presence of both Lgr5- and DcamKL-1-positive cells in our tissue-engineered intestine reinforces our observation that engineered intestine is very similar to native intestine. Presence of those cells may be crucial for the normal homeostasis of the tissue for the lifetime of a patient in a clinical setting, and to exceed the current survival rates of nonautologous intestinal transplanted grafts. The presence of those putative stem cells also gives hope that the tissue would be able to repair itself in case of damage.

Recent experiments using isolated Lgr5-positive cells demonstrated that those cells were able to proliferate and form cyst-like structures with a differentiated epithelium in vitro. ${ }^{18}$ In order to achieve the development of crypt/villus structures from those isolated cells, the microenvironment of the cells was artificially recreated by culturing them in Matrigel with the addition of Wnt agonist R-spondin 1, EGF, and Noggin. ${ }^{18}$ However, this technique, although promising, has various limitations that will need to be addressed before translation to human therapy. First, the sorting process to purify single-cell populations is somewhat harsh and timeintensive. This allows the growth of only $6 \%$ of the cells, and their development was limited to a few crypt-villus structures. It may be difficult to scale up sorted single cells for autologous human therapy. Moreover, prolonged culture could result in transformation of the cells leading to either loss of function or tumorigenic potential. ${ }^{35}$ Finally, implantation of a stock cell line or nonautologous cultured cells might require immunosuppression. The generation of TESI with our multicellular approach required no in vitro culture, limiting the associated risk of cell transformation. However, most importantly, maintaining the epithelial-mesenchymal relationship allows a more rapid and robust growth with large amount of mucosa and a higher success rate of mucosal generation. Indeed, we were able to generate a new intestinal mucosa demonstrating epithelial differentiation in $86 \%$ of our implants. At 6 weeks, the resulting tissues contained in average 3 times the initial implanted number of cells. Moreover, this approach also provides the mesenchymal components (i.e., muscularis, nerves, and blood supply) necessary for functional intestine that single-cell approaches currently lack.

Transition to the mouse model is a very exciting advance, as it will allow us to further investigate the molecular and cellular mechanisms involved during the growth of tissueengineered intestine. A better understanding of those processes is crucial for a successful, safe, and optimized transition to human therapy.

\section{Acknowledgments}

This study was supported by grants from the California Institute for Regenerative Medicine (\#RN2-00946-1) (TG201168) and the Saban Research Institute.

\section{Disclosure Statement}

No competing financial interests exist.

\section{References}

1. Reyes, J., Bueno, J., Kocoshis, S., Green, M., Abu-Elmagd, K., Furukawa, H., et al. Current status of intestinal transplantation in children. J Pediatr Surg 33, 243, 1998.

2. Grant, D., Abu-Elmagd, K., Reyes, J., Tzakis, A., Langnas, A., Fishbein, T., et al. 2003 report of the intestine transplant registry: a new era has dawned. Ann Surg 241, 607, 2005.

3. Sudan, D., Thompson, J., Botha, J., Grant, W., Antonson, D., Raynor, S., et al. Comparison of intestinal lengthening procedures for patients with short bowel syndrome. Ann Surg 246, 593; discussion 4, 2007.

4. Nightingale, J.M., and Lennard-Jones, J.E. The short bowel syndrome: what's new and old? Dig Dis 11, 12, 1993.

5. Ladefoged, K., Hessov, I., and Jarnum, S. Nutrition in shortbowel syndrome. Scand J Gastroenterol Suppl 216, 122, 1996.

6. Spencer, A.U., Neaga, A., West, B., Safran, J., Brown, P., Btaiche, I., et al. Pediatric short bowel syndrome: redefining predictors of success. Ann Surg 242, 403; discussion 9, 2005.

7. Stollman, N.H., Neustater, B.R., and Rogers, A.I. Shortbowel syndrome. Gastroenterologist 4, 118, 1996.

8. Thompson, J.S. Management of the short bowel syndrome. Gastroenterol Clin North Am 23, 403, 1994.

9. Byrne, T.A., Nompleggi, D.J., and Wilmore, D.W. Advances in the management of patients with intestinal failure. Transplant Proc 28, 2683, 1996.

10. Wales, P.W., de Silva, N., Kim, J., Lecce, L., To, T., and Moore, A. Neonatal short bowel syndrome: populationbased estimates of incidence and mortality rates. J Pediatr Surg 39, 690, 2004.

11. Schalamon, J., Mayr, J.M., and Hollwarth, M.E. Mortality and economics in short bowel syndrome. Best Pract Res Clin Gastroenterol 17, 931, 2003.

12. Kato, T., Tzakis, A.G., Selvaggi, G., Gaynor, J.J., David, A.I., Bussotti, A., et al. Intestinal and multivisceral transplantation in children. Ann Surg 243, 756; discussion 64, 2006.

13. Sangiorgi, E., and Capecchi, M.R. Bmi1 is expressed in vivo in intestinal stem cells. Nat Genet 40, 915, 2008.

14. Barker, N., van Es, J.H., Kuipers, J., Kujala, P., van den Born, M., Cozijnsen, M., et al. Identification of stem cells in small intestine and colon by marker gene Lgr5. Nature 449, 1003, 2007. 
15. Zhu, L., Gibson, P., Currle, D.S., Tong, Y., Richardson, R.J., Bayazitov, I.T., et al. Prominin 1 marks intestinal stem cells that are susceptible to neoplastic transformation. Nature 457, 603, 2009.

16. May, R., Riehl, T.E., Hunt, C., Sureban, S.M., Anant, S., and Houchen, C.W. Identification of a novel putative gastrointestinal stem cell and adenoma stem cell marker, doublecortin and CaM kinase-like-1, following radiation injury and in adenomatous polyposis coli/multiple intestinal neoplasia mice. Stem Cells 26, 630, 2008.

17. Asai, R., Okano, H., and Yasugi, S. Correlation between Musashi-1 and c-hairy-1 expression and cell proliferation activity in the developing intestine and stomach of both chicken and mouse. Dev Growth Differ 47, 501, 2005.

18. Sato, T., Vries, R.G., Snippert, H.J., van de Wetering, M., Barker, N., Stange, D.E., et al. Single Lgr5 stem cells build crypt-villus structures in vitro without a mesenchymal niche. Nature 459, 262, 2009.

19. May, R., Sureban, S.M., Hoang, N., Riehl, T.E., Lightfoot, S.A., Ramanujam, R., et al. Doublecortin and CaM kinaselike-1 and leucine-rich-repeat-containing G-protein-coupled receptor mark quiescent and cycling intestinal stem cells, respectively. Stem Cells 27, 2571, 2009.

20. Grikscheit, T., Ochoa, E.R., Srinivasan, A., Gaissert, H., and Vacanti, J.P. Tissue-engineered esophagus: experimental substitution by onlay patch or interposition. J Thorac Cardiovasc Surg 126, 537, 2003.

21. Grikscheit, T., Srinivasan, A., and Vacanti, J.P. Tissueengineered stomach: a preliminary report of a versatile in vivo model with therapeutic potential. J Pediatr Surg 38, 1305, 2003.

22. Grikscheit, T.C., Ochoa, E.R., Ramsanahie, A., Alsberg, E., Mooney, D., Whang, E.E., et al. Tissue-engineered large intestine resembles native colon with appropriate in vitro physiology and architecture. Ann Surg 238, 35, 2003.

23. Grikscheit, T.C., Ogilvie, J.B., Ochoa, E.R., Alsberg, E., and Mooney, D., Vacanti, J.P. Tissue-engineered colon exhibits function in vivo. Surgery 132, 200, 2002.

24. Grikscheit, T.C., Siddique, A., Ochoa, E.R., Srinivasan, A., Alsberg, E., Hodin, R.A., et al. Tissue-engineered small intestine improves recovery after massive small bowel resection. Ann Surg 240, 748, 2004.

25. Grikscheit, T.C., and Vacanti, J.P. The history and current status of tissue engineering: the future of pediatric surgery. J Pediatr Surg 37, 277, 2002.

26. Ramsanahie, A., Duxbury, M.S., Grikscheit, T.C., Perez, A., Rhoads, D.B., Gardner-Thorpe, J., et al. Effect of GLP-2 on mucosal morphology and SGLT1 expression in tissueengineered neointestine. Am J Physiol Gastrointest Liver Physiol 285, G1345, 2003.
27. Sala, F.G., Kunisaki, S.M., Ochoa, E.R., Vacanti, J., and Grikscheit, T.C. Tissue-engineered small intestine and stomach form from autologous tissue in a preclinical large animal model. J Surg Res 156, 205, 2009.

28. Chen, D.C., Agopian, V.G., Avansino, J.R., Lee, J.K., Farley, S.M., and Stelzner, M. Optical tissue window: a novel model for optimizing engraftment of intestinal stem cell organoids. J Surg Res 134, 52, 2006.

29. Wright, D.E., Cheshier, S.H., Wagers, A.J., Randall, T.D., Christensen, J.L., and Weissman, I.L. Cyclophosphamide/ granulocyte colony-stimulating factor causes selective mobilization of bone marrow hematopoietic stem cells into the blood after M phase of the cell cycle. Blood 97, 2278, 2001.

30. Stappenbeck, T.S., and Miyoshi, H. The role of stromal stem cells in tissue regeneration and wound repair. Science 324, 1666, 2009.

31. Dekaney, C.M., Gulati, A.S., Garrison, A.P., Helmrath, M.A., and Henning, S.J. Regeneration of intestinal stem/progenitor cells following doxorubicin treatment of mice. Am J Physiol Gastrointest Liver Physiol 297, G461, 2009.

32. Andoh, A., Bamba, S., Brittan, M., Fujiyama, Y., and Wright, N.A. Role of intestinal subepithelial myofibroblasts in inflammation and regenerative response in the gut. Pharmacol Ther 114, 94, 2007.

33. Ramalho-Santos, M., Melton, D.A., and McMahon, A.P. Hedgehog signals regulate multiple aspects of gastrointestinal development. Development 127, 2763, 2000.

34. Sancho, E., Batlle, E., and Clevers, H. Signaling pathways in intestinal development and cancer. Annu Rev Cell Dev Biol 20, 695, 2004.

35. Mitalipova, M.M., Rao, R.R., Hoyer, D.M., Johnson, J.A., Meisner, L.F., Jones, K.L., et al. Preserving the genetic integrity of human embryonic stem cells. Nat Biotechnol 23, 19, 2005.

Address correspondence to: Tracy C. Grikscheit, M.D. Saban Research Institute Childrens Hospital Los Angeles 4650 W. Sunset Blvd MS\#100 Los Angeles, CA 900027

E-mail: tgrikscheit@chla.usc.edu

Received: September 24, 2010 Accepted: March 10, 2011

Online Publication Date: April 25, 2011 